\title{
G-Doob-Meyer Decomposition and Its Applications in Bid-Ask Pricing for Derivatives under Knightian Uncertainty
}

\author{
Wei Chen \\ School of Economics, Shandong University, Jinan 250100, China \\ Correspondence should be addressed to Wei Chen; weichen@sdu.edu.cn
}

Received 20 April 2015; Revised 23 July 2015; Accepted 9 August 2015

Academic Editor: Jafar Biazar

Copyright ( $) 2015$ Wei Chen. This is an open access article distributed under the Creative Commons Attribution License, which permits unrestricted use, distribution, and reproduction in any medium, provided the original work is properly cited.

\begin{abstract}
The target of this paper is to establish the bid-ask pricing framework for the American contingent claims against risky assets with G-asset price systems on the financial market under Knightian uncertainty. First, we prove G-Dooby-Meyer decomposition for G-supermartingale. Furthermore, we consider bid-ask pricing American contingent claims under Knightian uncertainty, by using G-Dooby-Meyer decomposition; we construct dynamic superhedge strategies for the optimal stopping problem and prove that the value functions of the optimal stopping problems are the bid and ask prices of the American contingent claims under Knightian uncertainty. Finally, we consider a free boundary problem, prove the strong solution existence of the free boundary problem, and derive that the value function of the optimal stopping problem is equivalent to the strong solution to the free boundary problem.
\end{abstract}

\section{Introduction}

The earliest and one of the most penetrating analyses on the pricing of the American option is by McKean [1]. There the problem of pricing the American option is transformed into a Stefan or free boundary problem. Solving the latter, McKean writes the American option price explicitly up to knowing a certain function, the optimal stopping boundary.

Bensoussan [2] presents a rigorous treatment for American contingent claims that can be exercised at any time before or at maturity. He adapts the Black and Scholes [3] methodology of duplicating the cash flow from such a claim to this situation by skillfully managing a self-financing portfolio that contains only the basic instruments of the market, that is, the stocks and the bond, and that entails no arbitrage opportunities before exercise. Bensoussan shows that the pricing of such claims is indeed possible and characterized the exercise time by means of an appropriate optimal stopping problem. In the study of the latter, Bensoussan employs the so-called "penalization method," which forces rather stringent boundedness and regularity conditions on the payoff from the contingent claim.

From the theory of optimal stopping, it is well known that the value process of the optimal stopping problem can be characterized as the smallest supermartingale majorant to the stopping reward. Based on the Doob-Meyer decomposition for the supermartingale, a "martingale" treatment of the optimal stopping problem is used for handling pricing of the American option by Karatzas [4] and El Karoui and Karatzas $[5,6]$.

The Doob decomposition theorem was proved by and is named for Doob [7]. The analogous theorem in the continuous time case is the Doob-Meyer decomposition theorem proved by Meyer in $[8,9]$. For the pricing American option problem in incomplete market, Kramkov [10] constructs the optional decomposition of supermartingale with respect to a family of equivalent local martingale measures. He calls such a representation optional because, in contrast to the Doob-Meyer decomposition, it generally exists only with an adapted (optional) process C. He applies this decomposition to the problem of hedging European and American style contingent claims in the setting of incomplete security markets. Using the optional decomposition, Frey [11] considers construction of superreplication strategies via optimal stopping which is similar to the optimal stopping problem that arises in the pricing of American-type derivatives on a family of probability space with equivalent local martingale measures.

For the realistic financial market, the asset price in the future is uncertain, the probability distribution of the asset 
price in the future is unknown, which is called Knightian uncertainty [12]. The probability distribution of the nature state in the future is unknown; investors have uncertain subjective belief, which makes their consumption and portfolio choice decisions uncertain and leads the uncertain asset price in the future. Pricing contingent claims against such assets under Knightian uncertainty is an open problem. Peng in $[13,14]$ constructs $G$ frame work which is an analysis tool for nonlinear system and is applied in pricing European contingent claims under volatility uncertainty $[15,16]$.

The target of this paper is to establish the bid-ask pricing framework for the American contingent claims against risky assets with G-asset price systems (see [17]) on the financial market under Knightian uncertainty. Firstly, on sublinear expectation space, by using potential theory and sublinear expectation theory we construct G-Doob-Meyer decomposition for G-supermartingale, that is, a right continuous Gsupermartingale could be decomposed as a G-martingale and a right continuous increasing process and the decomposition is unique. Second, we define bid and ask prices of the American contingent claim against the assets with G-asset price systems and apply the G-Doob-Meyer decomposition to prove that the bid and ask prices of American contingent claims under Knightian uncertainty could be described by the optimal stopping problems. Finally, we present a free boundary problem, and by using the penalization technique (see [18]) we derive that if there exists strong supersolution to the free boundary problem, then the strong solution to the free boundary problem exists. And by using truncation and regularization technique, we prove that the strong solution to the free boundary problem is the value function of the optimal stopping problem which is corresponding with pricing problem of the American contingent claim under Knightian uncertainty.

The rest of this paper is organized as follows. In Section 2, we give preliminaries for the sublinear expectation theory. In Section 3 we prove G-Doob-Meyer decomposition for Gsupermartingale. In Section 4, using G-Doob-Meyer decomposition, we construct dynamic superhedge strategies for the optimal stopping problem and prove that the solution of the optimal stopping problem is the bid and ask prices of the American contingent claims under Knightian uncertainty. In Section 5, we consider a free boundary problem, prove the strong solution existence of the free boundary problem, and derive that the solution of the optimal stopping problem is equivalent to the strong solution to the free boundary problem.

\section{Preliminaries}

Let $\Omega$ be a given set and let $\mathscr{H}$ be a linear space of real valued functions defined on $\Omega$ containing constants. The space $\mathscr{H}$ is also called the space of random variables.

Definition 1. A sublinear expectation $\widehat{E}$ is a functional $\widehat{E}$ : $\mathscr{H} \rightarrow R$ satisfying (i) monotonicity:

$$
\widehat{E}[X] \geq \widehat{E}[Y] \quad \text { if } X \geq Y,
$$

(ii) constant preserving:

$$
\widehat{E}[c]=c \quad \text { for } c \in R,
$$

(iii) subadditivity: for each $X, Y \in \mathscr{H}$,

$$
\widehat{E}[X+Y] \leq \widehat{E}[X]+\widehat{E}[Y],
$$

(iv) positive homogeneity:

$$
\widehat{E}[\lambda X]=\lambda \widehat{E}[X] \quad \text { for } \lambda \geq 0 .
$$

The triple $(\Omega, \mathscr{H}, \widehat{E})$ is called a sublinear expectation space.

In this section, we mainly consider the following type of sublinear expectation spaces $(\Omega, \mathscr{H}, \widehat{E})$ : if $X_{1}, X_{2}, \ldots, X_{n} \in$ $\mathscr{H}$ then $\varphi\left(X_{1}, X_{2}, \ldots, X_{n}\right) \in \mathscr{H}$ for $\varphi \in C_{b \text {,Lip }}\left(R^{n}\right)$, where $C_{b, \text { Lip }}\left(R^{n}\right)$ denotes the linear space of functions $\phi$ satisfying

$$
|\phi(x)-\phi(y)| \leq C\left(1+|x|^{m}+|y|^{m}\right)|x-y|
$$

for $x, y \in R$, some $C>0, m \in N$ is depending on $\phi$.

For each fixed $p \geq 1$, we take $\mathscr{H}_{0}^{p}=\left\{X \in \mathscr{H}, \widehat{E}\left[|X|^{p}\right]=\right.$ $0\}$ as our null space and denote $\mathscr{H} / \mathscr{H}_{0}^{p}$ as the quotient space. We set $\|X\|_{p}:=\left(\widehat{E}\left[|X|^{p}\right]\right)^{1 / p}$ and extend $\mathscr{H} / \mathscr{H}_{0}^{p}$ to its completion $\widehat{\mathscr{H}}_{p}$ under $\|\cdot\|_{p}$. Under $\|\cdot\|_{p}$ the sublinear expectation $\widehat{E}$ can be continuously extended to the Banach space $\left(\widehat{\mathscr{H}}_{p},\|\cdot\|_{p}\right)$. Without loss generality, we denote the Banach space $\left(\widehat{\mathscr{H}}_{p},\|\cdot\|_{p}\right)$ as $L_{G}^{p}(\Omega, \mathscr{H}, \widehat{E})$. For the G-frame work, we refer to $[13,14]$.

In this paper we assume that $\mu, \bar{\mu}, \underline{\sigma}$, and $\bar{\sigma}$ are positive constants such that $\underline{\mu} \leq \bar{\mu}$ and $\underline{\sigma} \leq \overline{\bar{\sigma}}$.

Definition 2. Let $X_{1}$ and $X_{2}$ be two random variables in a sublinear expectation space $(\Omega, \mathscr{H}, \widehat{E}) ; X_{1}$ and $X_{2}$ are called identically distributed, denoted by $X_{1} \stackrel{d}{=} X_{2}$ if

$$
\widehat{E}\left[\phi\left(X_{1}\right)\right]=\widehat{E}\left[\phi\left(X_{2}\right)\right] \quad \forall \phi \in C_{b, \text { Lip }}\left(R^{n}\right) .
$$

Definition 3. In a sublinear expectation space $(\Omega, \mathscr{H}, \widehat{E})$, a random variable $Y$ is said to be independent of another random variable $X$, if

$$
\widehat{E}[\phi(X, Y)]=\widehat{E}\left[\left.\widehat{E}[\phi(x, Y)]\right|_{x=X}\right] .
$$

Definition 4 (G-normal distribution). A random variable $X$ on a sublinear expectation space $(\Omega, \mathscr{H}, \widehat{E})$ is called G-normal distributed if

$$
a X+b \bar{X}=\sqrt{a^{2}+b^{2}} X \quad \text { for } a, b \geq 0,
$$

where $\bar{X}$ is an independent copy of $X$. 
We denote by $S(d)$ the collection of all $d \times d$ symmetric matrices. Let $X$ be G-normal distributed random vectors on $(\Omega, \mathscr{H}, \widehat{E})$; we define the following sublinear function:

$$
G(A):=\frac{1}{2} \widehat{E}[\langle A X, X\rangle], \quad A \in S(d) .
$$

Remark 5. For a random variable $X$ on the sublinear space $(\Omega, \mathscr{H}, \widehat{E})$, there are four typical parameters to character $X$ :

$$
\begin{aligned}
& \bar{\mu}_{X}=\widehat{E} X, \\
& \underline{\mu}_{X}=-\widehat{E}[-X], \\
& \bar{\sigma}_{X}^{2}=\widehat{E} X^{2}, \\
& \underline{\sigma}_{X}^{2}=-\widehat{E}\left[-X^{2}\right],
\end{aligned}
$$

where $\left[\underline{\mu}_{X}, \bar{\mu}_{X}\right]$ and $\left[\underline{\sigma}_{X}^{2}, \bar{\sigma}_{X}^{2}\right]$ describe the uncertainty of the mean and the variance of $X$, respectively.

It is easy to check that if $X$ is G-normal distributed, then

$$
\bar{\mu}_{X}=\widehat{E} X=\underline{\mu}_{X}=-\widehat{E}[-X]=0,
$$

and we denote the G-normal distribution as $N\left(\{0\},\left[\underline{\sigma}^{2}, \bar{\sigma}^{2}\right]\right)$. If $X$ is maximally distributed, then

$$
\bar{\sigma}_{X}^{2}=\widehat{E} X^{2}=\underline{\sigma}_{X}^{2}=-\widehat{E}\left[-X^{2}\right]=0,
$$

and we denote the maximal distribution (see [14]) as $N([\mu, \bar{\mu}],\{0\})$.

Let $\mathscr{F}$ as Borel field subsets of $\Omega$. We are given a family $\left\{\mathscr{F}_{t}\right\}_{t \in R_{+}}$of Borel subfields of $\mathscr{F}$, such that

$$
\mathscr{F}_{s} \subset \mathscr{F}_{t}, \quad s<t .
$$

Definition 6. We call $\left(X_{t}\right)_{t \in R}$ a $d$-dimensional stochastic process on a sublinear expectation space $\left(\Omega, \mathscr{H}, \widehat{E}, \mathscr{F},\{\mathscr{F}\}_{t \in R_{+}}\right)$, if, for each $t \in R, X_{t}$ is a $d$-dimensional random vector in $\mathscr{H}$.

Definition 7. Let $\left(X_{t}\right)_{t \in R}$ and $\left(Y_{t}\right)_{t \in R}$ be $d$-dimensional stochastic processes defined on a sublinear expectation space $\left(\Omega, \mathscr{H}, \widehat{E}, \mathscr{F},\{\mathscr{F}\}_{t \in R_{+}}\right)$, for each $\underline{t}=\left(t_{1}, t_{2}, \ldots, t_{n}\right) \in \mathscr{T} ;$

$$
F_{\underline{t}}^{X}[\varphi]:=\widehat{E}\left[\varphi\left(X_{\underline{t}}\right)\right], \quad \forall \varphi \in C_{l, \text { Lip }}\left(R^{n \times d}\right)
$$

is called the finite dimensional distribution of $X_{t} . X$ and $Y$ are said to be identically distributed, that is, $X \stackrel{d}{=} Y$, if

$$
F_{\underline{t}}^{X}[\varphi]=F_{\underline{t}}^{Y}[\varphi], \quad \forall \underline{t} \in \mathscr{T}, \forall \varphi \in C_{l, \text { Lip }}\left(R^{n \times d}\right),
$$

where $\mathscr{T}:=\left\{\underline{t}=\left(t_{1}, t_{2}, \ldots, t_{n}\right): \forall n \in N, t_{i} \in R, t_{i} \neq t_{j}, 0 \leq\right.$ $i, j \leq n, i \neq j\}$.

Definition 8. A process $\left(B_{t}\right)_{t \geq 0}$ on the sublinear expectation space $\left(\Omega, \mathscr{H}, \widehat{E}, \mathscr{F},\{\mathscr{F}\}_{t \in R_{+}}\right)$is called a G-Brownian motion if the following properties are satisfied:

(i) $B_{0}(\omega)=0$;

(ii) For each $t, s>0$, the increment $B_{t+s}-B_{t}$ is G-normal distributed by $N\left(\{0\},\left[s \sigma^{2}, s \bar{\sigma}^{2}\right]\right)$ and is independent of $\left(B_{t_{1}}, B_{t_{2}}, \ldots, B_{t_{n}}\right)$, for each $n \in N$ and $t_{1}, t_{2}, \ldots, t_{n} \in$ $(0, t]$.

From now on, the stochastic processes we will consider in the rest of this paper are all in the sublinear space $\left(\Omega, \mathscr{H}, \widehat{E}, \mathscr{F},\{\mathscr{F}\}_{t \in R_{+}}\right)$.

\section{G-Doob-Meyer Decomposition for G-Supermartingale}

Definition 9. A G-supermartingale (resp., G-submartingale) is a real valued process $\left\{X_{t}\right\}$, well adapted to the $\mathscr{F}_{t}$ family, such that

(i) $\widehat{E}\left[\left|X_{t}\right|\right]<\infty \quad \forall t \in R_{+}$,

(ii) $\widehat{E}\left[X_{t+s} \mid \mathscr{F}_{s}\right] \leq($ resp. $\geq) X_{s}$

$$
\forall t \in R_{+}, \forall s \in R_{+} .
$$

If equality holds in (ii), the process is a G-martingale.

We will consider right continuous G-supermartingales; then if $\left\{X_{t}\right\}$ is right continuous G-supermartingale, (ii) in (16) holds with $\mathscr{F}_{t}$ replaced by $\mathscr{F}_{t+}$.

Definition 10. Let $A$ be an event in $\mathscr{F}_{t+}$; one defines capacity of $A$ as

$$
c(A)=\widehat{E}\left[I_{A}\right],
$$

where $I_{A}$ is indicator function of event $A$.

Definition 11. Process $X_{t}$ and $Y_{t}$ are adapted to the filtration $\mathscr{F}_{t}$. One calls $Y_{t}$ equivalent to $X_{t}$, if and only if

$$
c\left(Y_{t} \neq X_{t}\right)=0 .
$$

For a right continuous G-supermartingale $\left\{X_{t}\right\}$ with $\widehat{E}\left[X_{t}\right]$ is right continuous function of $t$; we can find a right continuous G-supermartingale $\left\{Y_{t}\right\}$ equivalent to $\left\{X_{t}\right\}$ by defining 


$$
Y_{t}(\omega):= \begin{cases}X_{t+}(\omega)=\lim _{s \downarrow t} X_{s}(\omega), & \text { for any } \omega \in \Omega \text { such that the limit exits } \\ 0, & \text { otherwise. }\end{cases}
$$

Without loss generality, we denote $\mathscr{F}_{t}=\mathscr{F}_{t+}$.

Definition 12. For a positive constant $T$, one defines stop time $\tau$ in $[0, T]$ as a positive, random variable $\tau(\omega)$ such that $\{\tau \leq$ $T\} \in \mathscr{F}_{T}$.

In $[19,20]$, authors discuss the definition of stop time and its related theory in $\mathrm{G}$ frame work.

Let $\left\{X_{t}\right\}$ be a right continuous $\mathrm{G}$-supermartingale, denote $X_{\infty}$ as the last element of the process $X_{t}$, and then the process $\left\{X_{t}\right\}_{0 \leq t \leq \infty}$ is a G-supermartingale.

Definition 13. A right continuous increasing process is a well adapted stochastic process $\left\{A_{t}\right\}$ such that

(i) $A_{0}=0$ a.s,

(ii) for almost every $\omega$, the function $t \rightarrow A_{t}(\omega)$ is positive, increasing, and right continuous. Let $A_{\infty}(\omega):=$ $\lim _{t \rightarrow \infty} A_{t}(\omega)$; one will say that the right continuous increasing process is integrable if $\widehat{E}\left[A_{\infty}\right]<\infty$.

Definition 14. An increasing process $A$ is called natural if for every bounded, right continuous G-martingale $\left\{M_{t}\right\}_{0 \leq t<\infty}$ we have

$$
\widehat{E}\left[\int_{(0, t]} M_{s} d A_{s}\right]=\widehat{E}\left[\int_{(0, t]} M_{s^{-}} d A_{s}\right]
$$

for every $0<t<\infty$.

Lemma 15. If $A$ is an increasing process and $\left\{M_{t}\right\}_{0 \leq t<\infty}$ is bounded, right continuous G-martingale, then

$$
\widehat{E}\left[M_{t} A_{t}\right]=\widehat{E}\left[\int_{(0, t]} M_{s} d A_{s}\right]
$$

In particular, condition (20) in Definition 14 is equivalent to

$$
\widehat{E}\left[M_{t} A_{t}\right]=\widehat{E}\left[\int_{(0, t]} M_{s_{-}} d A_{s}\right]
$$

Proof. For a partition $\Pi=\left\{t_{0}, t_{1}, \ldots, t_{n}\right\}$ of $[0, t]$, with $0=$ $t_{0} \leq t_{1} \leq \cdots \leq t_{n}=t$, we define

$$
M_{s}^{\Pi}=\sum_{k=1}^{n} M_{t_{n}} I_{\left(t_{k-1}, t_{k}\right]}(s)
$$

Since $M$ is G-martingale

$$
\begin{aligned}
& \widehat{E}\left[\int_{(0, t]} M_{s}^{\Pi} d A_{s}\right]=\widehat{E}\left[\sum_{k=1}^{n} M_{t_{k}}\left(A_{t_{k}}-A_{t_{k-1}}\right)\right] \\
& \quad=\widehat{E}\left[\sum_{k=1}^{n} M_{t_{k}} A_{t_{k}}-\sum_{k=1}^{n-1} M_{t_{k+1}} A_{t_{k}}\right] \\
& =\widehat{E}\left[M_{t} A_{t}-\sum_{k=1}^{n-1}\left(M_{t_{k+1}}-M_{t_{k}}\right) A_{t_{k}}\right] \\
& =\widehat{E}\left[M_{t} A_{t}-\sum_{k=1}^{n-1}\left(M_{t_{k+1}}-M_{t_{k}}\right) A_{t_{k}}\right] \\
& =\widehat{E}\left[M_{t} A_{t}\right],
\end{aligned}
$$

and we finish the proof of the Lemma.

Definition 16. A positive right continuous G-supermartingale $\left\{Y_{t}\right\}$ with $\lim _{t \rightarrow \infty} Y_{t}(\omega)=0$ is called a potential.

Definition 17. For $a \in[0, \infty]$, a process $\left\{X_{t}, t \in[0, a]\right\}$ is said to be uniformly integrable on $[0, a]$ if

$$
\sup _{t \in[0, a]} \widehat{E}\left[\left|X_{t}\right| I_{\left|X_{t}\right|>x}\right] \longrightarrow 0, \quad \text { as } x \longrightarrow 0
$$

Definition 18. Let $a \in[0, \infty]$, and let $\left\{X_{t}\right\}$ be a right continuous process; we will say that it belongs to the class (GD) on this interval, if all the random variables $X_{T}$ are uniformly integrable and $T$ is stop time bounded by $a$. If $\left\{X_{t}\right\}$ belongs to the class (GD) on every interval $[0, a], a<\infty$, it will be said to belong locally to the class (GD).

If $\left\{A_{t}\right\}$ is an integrable right continuous, increasing process, then process $\left\{-A_{t}\right\}$ is a negative G-supermartingale, and $\left\{\widehat{E}\left[A_{\infty} \mid \mathscr{F}_{t}\right]-A_{t}\right\}$ is a potential of the class (GD), which we will call the potential generated by $\left\{A_{t}\right\}$.

Proposition 19. (1) Any right continuous G-martingale $\left\{X_{t}\right\}$ belongs locally to class (GD).

(2) Any right continuous G-supermartingale $\left\{X_{t}\right\}$, which is bounded from above, belongs locally to class (GD).

(3) Any right continuous supermartingale $\left\{X_{t}\right\}$, which belongs locally to class (GD) and is uniformly integrable, belongs to class (GD).

Proof. (1) If $a<\infty$ and $T$ is a stop time, $T \leq a$, then Gmartingale process $\left\{X_{t}\right\}$ has $X_{T}=\widehat{E}\left[X_{a} \mid \mathscr{F}_{T}\right]$. Hence

$$
\widehat{E}\left[\left|X_{T}\right| I_{\left\{\left|X_{T}\right|>n\right\}}\right] \leq \widehat{E}\left[\left|X_{a}\right| I_{\left\{\left|X_{T}\right|>n\right\}}\right] .
$$


As $n \cdot c\left(\left|X_{T}\right|>n\right) \leq \widehat{E}\left[\left|X_{T}\right|\right] \leq \widehat{E}\left[\left|X_{a}\right|\right]$, we have $c\left(\left|X_{T}\right|>n\right) \rightarrow 0$ as $n \rightarrow \infty$; then $\widehat{E}\left[\left|X_{a}\right| I_{\left\{\left|X_{T}\right|>n\right\}}\right] \leq$ $\left(\widehat{E}\left[\left|X_{d}\right|^{2}\right]\right)^{1 / 2}\left(c\left(I_{\left\{\left|X_{T}\right|>n\right\}}\right)\right)^{1 / 2} \rightarrow 0$ as $n \rightarrow \infty$, from which we prove (1).

(2) If $a<\infty$ and $T$ is a stop time, $T \leq a$, then G-supermartingale process $\left\{X_{t}\right\}$ has $X_{T} \geq \widehat{E}\left[X_{a} \mid \mathscr{F}_{T}\right]$. Suppose that $\left\{X_{t}\right\}$ is negative; then

$$
\widehat{E}\left[-X_{T} I_{\left\{X_{T}<-n\right\}}\right] \leq \widehat{E}\left[-X_{a} I_{\left\{X_{T}<-n\right\}}\right] ;
$$

we complete the proof of (2) by using similar argument in proof (1).

(3) $\left\{X_{t}\right\}$ is uniformly integrable; we set

$$
X_{t}=\widehat{E}\left[X_{\infty} \mid \mathscr{F}_{t}\right]+\left(X_{t}-\widehat{E}\left[X_{\infty} \mid \mathscr{F}_{t}\right]\right) .
$$

The first part on the right-hand of the above equation $\widehat{E}\left[X_{\infty} \mid \mathscr{F}_{t}\right]$ is a G-martingale and equivalent to a right continuous process, and from (1) we know that it belongs to class (GD). We denote the second part in the above equation as $\left\{Y_{t}\right\}$; it is a potential, that is, a positive right continuous G-supermartingale, and $\lim _{t \rightarrow \infty} Y_{t}(\omega)=0$ a.s. Next we will prove that $\left\{Y_{t}\right\}$ belongs to class (GD). Since both $\inf (T, a)$ and $\sup (T, a)$ are stop times

$$
\begin{aligned}
\widehat{E}\left[Y_{T} I_{\left\{Y_{T}>n\right\}}\right] & \leq \widehat{E}\left[Y_{T} I_{\left\{T \leq a, Y_{T}>n\right\}}\right]+\widehat{E}\left[Y_{T} I_{\{T>a\}}\right] \\
& \leq \widehat{E}\left[Y_{T} I_{\left\{T \leq a, Y_{T}>n\right\}}\right]+\widehat{E}\left[Y_{a}\right] .
\end{aligned}
$$

Consider that $\lim _{a \rightarrow \infty} \widehat{E}\left[Y_{a}\right]=0$ and $\left\{Y_{t}\right\}$ locally belongs to (GD); that is, $\lim _{n \rightarrow \infty} \widehat{E}\left[Y_{T} I_{\left\{T \leq a, Y_{T}>n\right\}}\right]=0$, which prove that

$$
\lim _{n \rightarrow \infty} \widehat{E}\left[Y_{T} I_{\left\{Y_{T}>n\right\}}\right]=0 .
$$

We complete the proof.

Lemma 20. Let $\left\{X_{t}\right\}$ be a right continuous G-supermartingale and $\left\{X_{t}^{n}\right\}$ a sequence of decomposed right continuous Gsupermartingale:

$$
X_{t}^{n}=M_{t}^{n}-A_{t}^{n},
$$

where $\left\{M_{t}^{n}\right\}$ is G-martingale and $\left\{A_{t}^{n}\right\}$ is right continuous increasing process. Suppose that, for each $t, X_{t}^{n}$ converge to $X_{t}$ in the $L_{G}^{1}(\Omega)$ topology, and $A_{t}^{n}$ are uniformly integrable in $n$. Then the decomposition problem is solvable for the $G$-supermartingale $\left\{X_{t}\right\}$; more precisely, there are a right continuous increasing process $\left\{A_{t}\right\}$ and a G-martingale $\left\{M_{t}\right\}$, such that $X_{t}=M_{t}-A_{t}$.

Proof. We denote by $w$ the weak topology $w\left(L_{G}^{1}(\Omega), L_{G}^{\infty}(\Omega)\right)$; a sequence of integrable random variables $f_{n}$ converges to a random variable $f$ in the $w$-topology, if and only if $f$ is integrable, and

$$
\lim _{n \rightarrow \infty} \widehat{E}\left[f_{n} g\right]=\widehat{E}[f g], \quad \forall g \in L_{G}^{\infty}(\Omega) .
$$

Since $A_{t}^{n}$ are uniformly integrable in $n$, by the properties of the sublinear expectation $\widehat{E}[\cdot]$ there exists a $w$-convergent subsequence $A_{t}^{n_{k}}$ converging in the $w$-topology to the random variables $A_{t}^{\prime}$, for all rational values of $t$. To simplify the notations, we will use $A_{t}^{n}$ converging to $A_{t}^{\prime}$ in the $w$-topology for all rational values of $t$. An integrable random variable $f$ is $\mathscr{F}_{t}$-measurable if and only if it is orthogonal to all bounded random variables $g$ such that $\widehat{E}\left[g \mid \mathscr{F}_{t}\right]=0$; it follows that $A_{t}^{\prime}$ is $\mathscr{F}_{t}$-measurable. For $s<t, s$ and $t$ rational,

$$
\widehat{E}\left[\left(A_{t}^{n}-A_{s}^{n}\right) I_{B}\right] \geq 0,
$$

where $B$ denote any $\mathscr{F}$ set.

As $X_{t}^{n}$ converge to $X_{t}$ in $L_{G}^{1}(\Omega)$ topology, which is in a stronger topology than $w$, the $M_{t}^{n}$ converge to random variables $M_{t}^{\prime}$ for $t$ rational, and the process $\left\{M_{t}^{\prime}\right\}$ is Gmartingale; then there is a right continuous G-martingale $\left\{M_{t}\right\}$, defined for all values of $t$, such that $c\left(M_{t} \neq M_{t}^{\prime}\right)=0$ for each rational $t$. We define $A_{t}=X_{t}+M_{t} ;\left\{A_{t}\right\}$ is a right continuous increasing process or at least becomes so after a modification on a set of measure zero. We complete the proof.

Lemma 21. Let $\left\{X_{t}\right\}$ be a potential and belong to class (GD). One considers the measurable, positive, and well-adapted processes $H=\left\{H_{t}\right\}$ with the property that the right continuous increasing processes

$$
A(H)=\left\{A_{t}(H, \omega)\right\}=\left\{\int_{0}^{t} H_{s}(\omega) d s\right\}
$$

are integrable, and the potentials $Y(H)=\left\{Y_{t}(H, \omega)\right\}$ they generate are majorized by $X_{t}$. Then, for each $t$, the random variables $A_{t}(H)$ of all such processes $A(H)$ are uniformly integrable.

Proof. It is sufficient to prove that the $A_{\infty}(H)$ are uniformly integrable.

(1) First we assume that $X_{t}$ is bounded by some positive constant $C$; then $\widehat{E}\left[A_{\infty}^{2}(H)\right] \leq 2 C^{2}$, and the uniform integrability follows.

We have that

$$
\begin{aligned}
A_{\infty}^{2} & (H, \omega) \\
& =2 \int_{0}^{\infty}\left[A_{\infty}(H, \omega)-A_{u}(H, \omega)\right] d A_{u}(H, \omega) \\
& =2 \int_{0}^{\infty}\left[A_{\infty}(H, \omega)-A_{u}(H, \omega)\right] H_{u}(\omega) d u .
\end{aligned}
$$

By using the subadditive property of the sublinear expectation $\widehat{E}$, we derive that

$$
\begin{aligned}
\widehat{E} & {\left[A_{\infty}^{2}(H, \omega)\right]=\widehat{E}\left[\widehat{E}\left[A_{\infty}^{2}(H, \omega) \mid \mathscr{F}_{t}\right]\right] } \\
& \leq 2 \widehat{E}\left[\int_{0}^{\infty} H_{u} \widehat{E}\left[A_{\infty}(H, \omega)-A_{u}(H, \omega) \mid \mathscr{F}_{u}\right] d u\right] \\
& =2 \widehat{E}\left[\int_{0}^{\infty} H_{u} Y_{u}(H) d u\right] \leq 2 C \widehat{E}\left[\int_{0}^{\infty} H_{u} d u\right] \\
& =2 C \widehat{E}\left[Y_{0}(H)\right] \leq 2 C^{2} .
\end{aligned}
$$


(2) In order to prove the general case, it will be enough to prove that any $H$ such that $Y(H)$ is majorized by $\left\{X_{t}\right\}$ is equal to a sum $H^{c}+H_{c}$, where (i) $A\left(H^{c}\right)$ generates a potential bounded by $c$, and (ii) $\widehat{E}\left[A_{\infty}\left[H_{c}\right]\right]$ is smaller than some number $\varepsilon_{c}$, independent of $H$, such that $\varepsilon_{c} \rightarrow 0$ as $c \rightarrow 0$. Define

$$
\begin{aligned}
H_{t}^{c}(\omega) & =H_{t}(\omega) I_{\left\{X_{t}(\omega) \in[0, c]\right\}}, \\
H_{c t} & =H_{t}-H_{t}^{c} .
\end{aligned}
$$

Set

$$
T^{c}(\omega)=\inf \left\{t: \text { such that } X_{t}(\omega) \geq c\right\}
$$

as $c$ goes to infinity $\lim _{c \rightarrow \infty} T^{c}(\omega)=\infty$; therefore $X_{T^{c}} \rightarrow 0$, and class $(\mathrm{GD})$ property implies that $\widehat{E}\left[X_{T^{c}}\right] \rightarrow 0 . T^{c}$ is a stop time, and $I_{\left\{X_{t}(\omega) \in[0, c]\right\}}=1$ before time $T^{c}$. Hence

$$
\begin{aligned}
\widehat{E}\left[A_{\infty}\left(H_{c}\right)\right] & =\widehat{E}\left[\int_{0}^{\infty} H_{u}\left(1-I_{\left\{X_{u}(\omega) \in[0, c]\right\}}\right)\right] d u \\
& \leq \widehat{E}\left[\int_{0}^{\infty} H_{u} d u\right] \\
& =\widehat{E}\left[A_{\infty}(H)-A_{T^{c}}(H)\right] \\
& =\widehat{E}\left[\widehat{E}\left[A_{\infty}(H)-A_{T^{c}}(H) \mid \mathscr{F}_{t}\right]\right] \\
& =\widehat{E}\left[Y_{T^{c}}(H)\right] \leq \widehat{E}\left[X_{T^{c}}(H)\right] \leq \varepsilon_{c},
\end{aligned}
$$

for large enough $c$,

from which we prove (ii). We will prove (i); first we prove that $Y\left(H^{c}\right)$ is bounded by $c$ :

$$
\begin{aligned}
Y_{t}\left(H^{c}\right) & =\widehat{E}\left[A_{\infty}\left(H^{c}\right)-A_{t}\left(H^{c}\right) \mid \mathscr{F}_{t}\right] \\
& =\widehat{E}\left[\int_{t}^{\infty} H_{u} I_{\left\{X_{u}(\omega) \in[0, c]\right\}} d u \mid \mathscr{F}_{t}\right] \\
& \leq \widehat{E}\left[\int_{S^{c}}^{\infty} H_{u} I_{\left\{X_{u}(\omega) \in[0, c]\right\}} d u \mid \mathscr{F}_{t}\right] \\
& =\widehat{E}\left[\widehat{E}\left[\int_{S^{c}}^{\infty} H_{u} I_{\left\{X_{u}(\omega) \in[0, c]\right\}} d u \mid \mathscr{F}_{S^{c}}\right] \mid \mathscr{F}_{t}\right] \\
& =\widehat{E}\left[Y_{S^{c}} \mid \mathscr{F}_{t}\right] \leq c,
\end{aligned}
$$

where we set

$$
S^{c}(\omega)=\inf \left\{t: \text { such that } X_{t}(\omega) \leq c\right\}
$$

and use

$$
\int_{t}^{S^{c}(\omega)} H_{u} I_{\left\{X_{u}(\omega) \in[0, c]\right\}} d u=0 .
$$

Inequality (40) holds for each $t$, for every rational $t$ and for every $t$ in consideration of the right continuity, which complete the proof.
Lemma 22. Let $\left\{X_{t}\right\}$ be a potential and belong to class (GD), $k$ is a positive number, define $Y_{t}=\widehat{E}\left[X_{t+k} \mid \mathscr{F}_{t}\right]$, and then $\left\{Y_{t}\right\}$ is a G-supermartingale. Denote by $\left\{p_{k} X_{t}\right\}$ a right continuous version of $\left\{Y_{t}\right\}$; then $\left\{p_{k} X_{t}\right\}$ is potential.

Use the same notations as in Lemma 21. Let $k$ be a positive number, and $H_{t, k}(\omega)=\left(X_{t}(\omega)-p_{k} X_{t}(\omega)\right) / k$. The process $H_{k}=\left\{H_{t, k}\right\}$ verifies the assumptions of Lemma 21, and their potentials increase to $\left\{X_{t}\right\}$ as $k \rightarrow 0$.

Proof. If $t<u$

$$
\begin{gathered}
\widehat{E}\left[\frac{1}{k}\left(\int_{0}^{u}\left[X_{s}-p_{k} X_{s}\right] d s-\int_{0}^{t}\left[X_{s}-p_{k} X_{s}\right] d s\right) \mid\right. \\
\left.\mathscr{F}_{t}\right]=\widehat{E}\left[\frac{1}{k} \int_{t}^{u}\left[X_{s}-p_{k} X_{s}\right] d s \mid \mathscr{F}_{t}\right] .
\end{gathered}
$$

For $s \geq t, \widehat{E}\left[p_{k} X_{s} \mid \mathscr{F}_{t}\right]=\widehat{E}\left[\widehat{E}\left[X_{s+k} \mid \mathscr{F}_{s}\right] \mid \mathscr{F}_{t}\right]=\widehat{E}\left[X_{s+k} \mid\right.$ $\left.\mathscr{F}_{t}\right]$. We have that

$$
\begin{aligned}
\widehat{E}\left[\frac{1}{k} \int_{t}^{u}\left[X_{s}-p_{k} X_{s}\right] d s \mid \mathscr{F}_{t}\right] \\
\geq \widehat{E}\left[\frac{1}{k} \int_{t}^{t+k} X_{s} d s \mid \mathscr{F}_{t}\right] \\
\quad-\widehat{E}\left[\frac{1}{k} \int_{u}^{u+k} X_{s} d s \mid \mathscr{F}_{t}\right],
\end{aligned}
$$

and, by the subadditive property of the sublinear expectation $\widehat{E}$, we derive that

$$
\begin{aligned}
& \widehat{E}\left[\frac{1}{k} \int_{t}^{t+k} X_{s} d s \mid \mathscr{F}_{t}\right]-\widehat{E}\left[\frac{1}{k} \int_{u}^{u+k} X_{s} d s \mid \mathscr{F}_{t}\right] \\
& \quad \geq \widehat{E}\left[\frac{1}{k} \int_{t}^{t+k} X_{s} d s \mid \mathscr{F}_{t}\right]-\frac{1}{k} \int_{u}^{u+k} \widehat{E}\left[X_{s} \mid \mathscr{F}_{t}\right] d s \\
& \quad \geq \widehat{E}\left[\frac{1}{k} \int_{t}^{t+k} X_{s} d s \mid \mathscr{F}_{t}\right]-X_{t} \\
& \geq-\widehat{E}\left[\frac{1}{k} \int_{t}^{t+k}\left(X_{t}-X_{s}\right) d s \mid \mathscr{F}_{t}\right] \\
& \quad \geq-\frac{1}{k} \int_{t}^{t+k} \widehat{E}\left[X_{t}-X_{s} \mid \mathscr{F}_{t}\right] d s \geq 0 .
\end{aligned}
$$

Hence, we derive that for any $u, t$ such that $u>t$

$$
\widehat{E}\left[\frac{1}{k} \int_{t}^{u}\left[X_{s}-p_{k} X_{s}\right] d s \mid \mathscr{F}_{t}\right] \geq 0 .
$$

If there exits $s_{0} \geq 0$ such that $(1 / k)\left[X_{s_{0}}-p_{k} X_{s_{0}}\right]<0$, the right continuous of $\left\{X_{t}\right\}$ implies that there exists $\delta>0$ such that $(1 / k)\left[X_{s}-p_{k} X_{s}\right]<0$ on the interval $\left[s_{0}, s_{0}+\delta\right]$. Thus

$$
\widehat{E}\left[\frac{1}{k} \int_{s_{0}}^{s_{0}+\delta}\left[X_{s}-p_{k} X_{s}\right] d s \mid \mathscr{F}_{s_{0}}\right]<0,
$$

which is contradiction; we prove that $\left(X_{t}(\omega)-p_{k} X_{t}(\omega)\right) / k$ is a positive, measurable, and well-adapted process. 
Since $\left\{X_{t}\right\}$ is right continuous G-supermartingale

$$
\begin{aligned}
\lim _{s \downarrow t} X_{s} & =X_{t}, \\
\lim _{k \downarrow 0} Y_{t}\left(H_{k}\right) & =\lim _{k \downarrow 0} \widehat{E}\left[\frac{1}{k} \int_{t}^{\infty}\left[X_{s}-p_{k} X_{s}\right] d s \mid \mathscr{F}_{t}\right] \\
& =\lim _{k \downarrow 0} \widehat{E}\left[\frac{1}{k} \int_{t}^{t+k} X_{s} d s \mid \mathscr{F}_{t}\right] \\
& =\widehat{E}\left[\lim _{k \downarrow 0} \frac{1}{k} \int_{t}^{t+k} X_{s} d s \mid \mathscr{F}_{t}\right]=X_{t} ;
\end{aligned}
$$

we finish the proof.

From Lemmas 20, 21, and 22 we can prove the following theorem.

Theorem 23. A potential $\left\{X_{t}\right\}$ belongs to class (GD) if and only if it is generated by some integrable right continuous increasing process.

Theorem 24 (G-Doob-Meyer's decomposition). (1) $\left\{X_{t}\right\}$ is a right continuous $G$-supermartingale if and only if it belongs to class (GD) on every finite interval. More precisely, $\left\{X_{t}\right\}$ is then equal to the difference of a G-martingal $M_{t}$ and a right continuous increasing process $A_{t}$ :

$$
X_{t}=M_{t}-A_{t} .
$$

(2) If the right continuous increasing process $A$ is natural, the decomposition is unique.

Proof. (1) The necessity is obvious. We will prove the sufficiency; we choose a positive number $a$ and define

$$
\begin{aligned}
& X_{t}^{\prime}(\omega):=X_{t}(\omega), \quad t \in[0, a], \\
& X_{t}^{\prime}(\omega):=X_{a}(\omega), \quad t>a ;
\end{aligned}
$$

the $\left\{X_{t}^{\prime}\right\}$ is a right continuous G-supermartingale of the class (GD), and by Theorem 23 there exists the following decomposition

$$
X_{t}^{\prime}=M_{t}^{\prime}-A_{t}^{\prime}
$$

where $\left\{M_{t}^{\prime}\right\}$ is a G-martingal and $\left\{A_{t}^{\prime}\right\}$ is a right continuous increasing process.

Let $a \rightarrow \infty$, as in Lemma 22 the expression of $Y_{t}\left(H_{k}\right)$ that $A_{t}^{\prime}$ depend only on the values of $\left\{X_{t}^{\prime}\right\}$ on intervals $[0, t+\varepsilon]$, with $\varepsilon$ small enough. As $a \rightarrow \infty$, they do not vary anymore once $a$ has reached values greater than $t$, as again Lemma 20; we finish the proof of the Theorem.

(2) Assume that $X$ admits both decompositions:

$$
X_{t}=M_{t}^{\prime}-A_{t}^{\prime}=M_{t}^{\prime \prime}-A_{t}^{\prime \prime},
$$

where $M_{t}^{\prime}$ and $M_{t}^{\prime \prime}$ are G-martingale and $A_{t}^{\prime}, A_{t}^{\prime \prime}$ are natural increasing process. We define

$$
\left\{C_{t}:=A_{t}^{\prime}-A_{t}^{\prime \prime}=M_{t}^{\prime}-M_{t}^{\prime \prime}\right\} .
$$

Then $\left\{C_{t}\right\}$ is a G-martingale, and, for every bounded and right continuous G-martingale $\left\{\xi_{t}\right\}$, from Lemma 15 we have

$$
\begin{aligned}
\widehat{E}\left[\xi_{t}\left(A_{t}^{\prime}-A_{t}^{\prime \prime}\right)\right] & =\widehat{E}\left[\int_{(0, t]} \xi_{s-} d C_{s}\right] \\
& =\lim _{n \rightarrow \infty} \sum_{k=1}^{m_{n}} \xi_{t_{j-1}^{n}}\left[C_{t_{j}^{(n)}}-C_{t_{j-1}^{(n)}}\right],
\end{aligned}
$$

where $\Pi_{n}=\left\{t_{0}^{(n)}, \ldots, t_{m_{n}}^{(n)}\right\}, n \geq 1$ is a sequence of partitions of $[0, t]$ with $\max _{1 \leq j \leq m_{n}}\left(t_{j}^{(n)}-t_{j-1}^{(n)}\right)$ converging to zero as $n \rightarrow$ $\infty$. Since $\xi$ and $C$ are both $G$-martingale, we have

$$
\widehat{E}\left[\xi_{t_{j-1}^{(n)}}\left(C_{t_{j}^{(n)}}-C_{t_{j-1}^{(n)}}\right)\right]=0,
$$

and thus $\widehat{E}\left[\xi_{t_{j-1}}\left(A_{t}^{\prime}-A_{t}^{\prime \prime}\right)\right]=0$.

For an arbitrary bonded random variable $\xi$, we can select $\left\{\xi_{t}\right\}$ to be a right continuous equivalent process of $\left\{\widehat{E}\left[\xi \mid \mathscr{F}_{t}\right]\right\}$, and we obtain that $\widehat{E}\left[\xi\left(A_{t}^{\prime}-A_{t}^{\prime \prime}\right)\right]=0$. We set $\xi=I_{A_{t}^{\prime} \neq A_{t}^{\prime \prime}}$; therefore $c\left(A_{t}^{\prime} \neq A_{t}^{\prime \prime}\right)=0$.

By Theorem 24 and G-martingale decomposition theorem in $[14,21]$, we have the following G-Doob-Meyer theorem.

Theorem 25. $\left\{X_{t}\right\}$ is a right continuous G-supermartingale; there exists a right continuous increasing process $A_{t}$ and adapted process $\eta_{t}$, such that

$$
X_{t}=\int_{0}^{t} \eta_{s} d B_{s}-A_{t}
$$

where $B_{t}$ is G-Brownian motion.

\section{Superhedging Strategies and Optimal Stopping}

4.1. Financial Model and G-Asset Price System. We consider a financial market with a nonrisky asset (bond) and a risky asset (stock) continuously trading in market. The price $P(t)$ of the bond is given by

$$
d P(t)=r P(t) d t \quad P(0)=1,
$$

where $r$ is the short interest rate; we assume a constant nonnegative short interest rate. We assume the risk asset with the G-asset price system $\left(\left(S_{u}\right)_{u \geq t}, \widehat{E}\right)$ (see [17]) on sublinear expectation space $\left(\Omega, \mathscr{H}, \widehat{E}, \mathscr{F},\left(\mathscr{F}_{t}\right)\right)$ under Knightian uncertainty, for given $t \in[0, T]$ and $x \in R$

$$
\begin{aligned}
d S_{u}^{t, x} & =S_{u}^{t, x} d B_{t}=S_{u}^{t, x}\left(d b_{t}+d \widehat{B}_{t}\right), \\
S_{t}^{t, x} & =x,
\end{aligned}
$$

where $B_{t}$ is the generalized G-Brownian motion. The uncertain volatility is described by the G-Brownian motion $\widehat{B}_{t}$. The uncertain drift $b_{t}$ can be rewritten as

$$
b_{t}=\int_{0}^{t} \mu_{u} d u,
$$


where $\mu_{t}$ is the asset return rate [22]. Then the uncertain risk premium of the G-asset price system

$$
\theta_{t}=\mu_{t}-r
$$

is uncertain and distributed by $N([\underline{\mu}-r, \bar{\mu}-r],\{0\})[22]$, where $r$ is the interest rate of the bond.

Define

$$
\widetilde{B}_{t}:=B_{t}-r t=b_{t}+\widehat{B}_{t}-r t ;
$$

we have the following G-Girsanov theorem (presented in [17, 23]).

Theorem 26 (G-Girsanov theorem). Assume that $\left(B_{t}\right)_{t \geq 0}$ is generalized G-Brownian motion on $\left(\Omega, \mathscr{H}, \widehat{E}, \mathscr{F}_{t}\right)$, and $\widetilde{B}_{t}$ is defined by (61); there exists G-expectation space $\left(\Omega, \mathscr{H}, E^{G}, \mathscr{F}_{t}\right)$ such that $\widetilde{B}_{t}$ is G-Brownian motion under the $G$-expectation $E^{G}$, and

$$
\begin{aligned}
\widehat{E}\left[\widehat{B}_{t}^{2}\right] & =E^{G}\left[\widetilde{B}_{t}^{2}\right], \\
-\widehat{E}\left[-\widehat{B}_{t}^{2}\right] & =-E^{G}\left[-\widetilde{B}_{t}^{2}\right] .
\end{aligned}
$$

By the G-Girsanov theorem, the G-asset price system (58) of the risky asset can be rewritten on $\left(\Omega, \mathscr{H}, E^{G}, \mathscr{F}_{t}\right)$ as follows:

$$
\begin{aligned}
d S_{u}^{t, x} & =S_{u}^{t, x}\left(r d t+d \widetilde{B}_{t}\right), \\
S_{t}^{t, x} & =x
\end{aligned}
$$

then by G-Itô formula we have

$$
S_{u}^{t, x}=x \exp \left(r(u-t)+\widetilde{B}_{u-t}-\frac{1}{2}\left(\left\langle\widetilde{B}_{u}\right\rangle-\left\langle\widetilde{B}_{t}\right\rangle\right)\right),
$$

$$
u>t \text {. }
$$

4.2. Construction of Superreplication Strategies via Optimal Stopping. We consider the following class of contingent claims.

Definition 27. One defines a class of contingent claims with the nonnegative payoff $\xi \in L_{G}^{2}\left(\Omega_{T}\right)$ having the following form:

$$
\xi=f\left(S_{T}^{t, x}\right)
$$

for some function $f: \Omega \rightarrow R$ such that the process

$$
f_{u}:=f\left(S_{u}^{t, x}\right)
$$

is bounded below and càdlàg.

We consider a contingent claim $\xi$ with payoff defined in Definition 27 written on the stockes $S_{t}$ with maturity $T$. We give definitions of superhedging (resp., subhedging) strategy and ask (resp., bid) price of the claim $\xi$.
Definition 28. (1) A self-financing superstrategy (resp. substrategy) is a vector process $(Y, \pi, C)($ resp., $(-Y, \pi, C))$, where $Y$ is the wealth process, $\pi$ is the portfolio process, and $C$ is the cumulative consumption process, such that

$$
\begin{gathered}
d Y_{t}=r Y_{t} d t+\pi_{t} d \widetilde{B}_{t}-d C_{t}, \\
\left(\text { resp. }-d Y_{t}=-r Y_{t} d t+\pi_{t} d \widetilde{B}_{t}-d C_{t}\right),
\end{gathered}
$$

where $C$ is an increasing, right continuous process with $C_{0}=$ 0 . The superstrategy (resp., substrategy) is called feasible if the constraint of nonnegative wealth holds

$$
Y_{t} \geq 0, \quad t \in[0, T] .
$$

(2) A superhedging (resp. subhedging) strategy against the contingent claim $\xi$ is a feasible self-financing superstrategy $(Y, \pi, C)$ (resp., substrategy $(-Y, \pi, C)$ ) such that $Y_{T}=\xi$ (resp., $\left.-Y_{T}=-\xi\right)$. We denote by $\mathscr{H}(\xi)$ (resp., $\left.\mathscr{H}^{\prime}(-\xi)\right)$ the class of superhedging (resp., subhedging) strategies against $\xi$, and if $\mathscr{H}(\xi)$ (resp., $\mathscr{H}^{\prime}(-\xi)$ ) is nonempty, $\xi$ is called superhedgeable (resp., subhedgeable).

(3) The ask-price $X(t)$ at time $t$ of the superhedgeable claim $\xi$ is defined as

$$
\begin{gathered}
X(t)=\inf \left\{x \geq 0: \exists\left(Y_{t}, \pi_{t}, C_{t}\right)\right. \\
\left.\in \mathscr{H}(\xi) \text { such that } Y_{t}=x\right\},
\end{gathered}
$$

and bid-price $X^{\prime}(t)$ at time $t$ of the subhedgeable claim $\xi$ is defined as

$$
\begin{aligned}
& X^{\prime}(t)=\sup \left\{x \geq 0: \exists\left(-Y_{t}, \pi_{t}, C_{t}\right)\right. \\
& \left.\in \mathscr{H}^{\prime}(-\xi) \text { such that }-Y_{t}=-x\right\} .
\end{aligned}
$$

Under uncertainty, the market is incomplete and the superhedging (resp., subhedging) strategy of the claim is not unique. The definition of the ask-price $X(t)$ implies that the ask-price $X(t)$ is the minimum amount of risk for the buyer to superhedging the claim; then it is coherent measure of risk of all superstrategies against the claim for the buyer. The coherent risk measure of all superstrategies against the claim can be regarded as the sublinear expectation of the claim; we have the following representation of bid-ask price of the claim via optimal stopping (Theorem 31).

Let $\left(\mathscr{G}_{t}\right)$ be a filtration on G-expectation space $(\Omega, \mathscr{H}$, $\left.E^{G}, \mathscr{F},\left(\mathscr{F}_{t}\right)_{t \geq 0}\right)$, and $\tau_{1}$ and $\tau_{2}$ be $\left(\mathscr{G}_{t}\right)$-stopping times such that $\tau_{1} \leq \tau_{2}$ a.s. We denote by $\mathscr{G}_{\tau_{1}, \tau_{2}}$ the set of all finite $\left(\mathscr{G}_{t}\right)$ stopping times $\tau$ with $\tau_{1} \leq \tau \leq \tau_{2}$.

For given $t \in[0, T]$ and $x \in R_{+}$, we define the function $V^{A m}:[0, T] \times \Omega \rightarrow R$ as the value function of the following optimal-stopping problem:

$$
\begin{aligned}
V^{A m}\left(t, S_{t}\right) & :=\sup _{v \in \mathscr{F}_{t, T}} E_{t}^{G}\left[f_{v}\right] \\
& =\sup _{v \in \mathscr{F}_{t, T}} E_{t}^{G}\left[f\left(S_{v}\right)\right] .
\end{aligned}
$$


Proposition 29. Consider two stopping times $\underline{\tau} \leq \bar{\tau}$ on filtration $\mathscr{F}$. Let $\left(f_{t}\right)_{t \geq 0}$ denote some adapted and RCLLstochastic process, which is bounded below. Then we have for two points $s, t \in[0, \bar{\tau}]$ and $s<t$

$$
\underset{\tau \in \mathscr{F}_{\underline{\tau}, \bar{\tau}}}{\operatorname{ess} \sup }\left\{E_{s}^{G}\left[f_{\tau}\right]\right\}=E_{s}^{G}\left[\underset{\tau \in \mathscr{F}_{\tau, \bar{\tau}}}{\operatorname{ess} \sup _{t}}\left\{E_{t}^{G}\left[f_{\tau}\right]\right\}\right] .
$$

Proof. By the consistent property of the conditional Gexpectation, for $\tau \in \mathscr{F}_{\underline{\tau}, \bar{\tau}}, s, t \in[0, \bar{\tau}]$, and $s<t$

$$
E_{s}^{G}\left[f_{\tau}\right]=E_{s}^{G}\left[E_{t}^{G}\left[f_{\tau}\right]\right] \leq E_{s}^{G}\left[\underset{\tau \in \mathscr{F}_{\tau, \bar{\tau}}}{\operatorname{ess} \sup _{t}}\left\{E_{t}^{G}\left[f_{\tau}\right]\right\}\right]
$$

thus we have

$$
\underset{\tau \in \mathscr{F}_{\underline{\tau}, \bar{\tau}}}{\operatorname{ess} \sup _{s}}\left\{E_{s}^{G}\left[f_{\tau}\right]\right\} \leq E_{s}^{G}\left[\underset{\tau \in \mathscr{F}_{\underline{\tau}, \bar{\tau}}}{\operatorname{ess} \sup _{t}}\left\{E_{\tau}^{G}\left[f_{\tau}\right]\right\}\right] .
$$

There exists a sequence $\left\{\tau_{n}\right\} \rightarrow \tau^{*} \in[\underline{\tau}, \bar{\tau}]$ as $n \rightarrow \infty$, such that

$$
\lim _{n \rightarrow \infty} E_{t}^{G}\left[f_{\tau_{n}}\right]=E_{t}^{G}\left[f_{\tau^{*}}\right]=\underset{\tau \in \mathscr{F}_{\underline{\tau}, \bar{\tau}}}{\operatorname{ess} \sup }\left\{E_{t}^{G}\left[f_{\tau}\right]\right\} ;
$$

notice that

$$
\begin{aligned}
& E_{s}^{G}\left[\underset{\tau \in \mathscr{F}_{\underline{\tau}, \bar{\tau}}}{\operatorname{ess} \sup _{t}}\left\{E_{t}^{G}\left[f_{\tau}\right]\right\}\right]=E_{s}^{G}\left[E_{t}^{G}\left[f_{\tau^{*}}\right]\right]=E_{s}^{G}\left[f_{\tau^{*}}\right] \\
& \quad \leq \underset{\tau \in \mathscr{F}_{\tau, \bar{\tau}}}{\operatorname{ess} \sup _{\tau,}}\left\{E_{s}^{G}\left[f_{\tau}\right]\right\} ;
\end{aligned}
$$

we prove the Proposition.

Proposition 30. The process $V^{A m}\left(t, S_{t}\right)_{0 \leq t \leq T}$ is a Gsupermartingale in $\left(\Omega, \mathscr{H}, E^{G}, \mathscr{F}, \mathscr{F}_{t}\right)$.

Proof. By Proposition 29, for $0 \leq s \leq t \leq T$

$$
E_{s}^{G}\left[\sup _{\nu \in \mathscr{F}_{t, T}} E_{t}^{G}\left[f\left(S_{\nu}\right)\right]\right]=\sup _{\nu \in \mathscr{F}_{t, T}} E_{s}^{G}\left[f\left(S_{\nu}\right)\right] .
$$

Since $\mathscr{F}_{t, T} \subseteq \mathscr{F}_{s, T}$, we have

$$
\sup _{v \in \mathscr{F}_{t, T}} E_{s}^{G}\left[f\left(S_{\nu}\right)\right] \leq \sup _{\nu \in \mathscr{F}_{s, T}} E_{s}^{G}\left[f\left(S_{\nu}\right)\right] .
$$

Thus, we derive that

$$
E_{s}^{G}\left[\sup _{\nu \in \mathscr{F}_{t, T}} E_{t}^{G}\left[f\left(S_{\nu}\right)\right]\right] \leq \sup _{\nu \in \mathscr{F}_{s, T}} E_{s}^{G}\left[f\left(S_{\nu}\right)\right] .
$$

We prove the Proposition.

Theorem 31. Assume that the financial market under uncertainty consists of the bond which has the price process satisfying (57) and risky assets with the price processes as the G-asset price systems (58) and can trade freely; the contingent claim $\xi$ which is written on the risky assets with the maturity $T>0$ has the class of the payoff defined in Definition 27, and the function $V^{A m}\left(t, S_{t}\right)$ is defined in (71). Then there exists a superhedging (resp., subhedging) strategy for $\xi$, such that the process $V=$ $\left(V_{t}\right)_{0 \leq t \leq T}$ defined by

$$
\begin{gathered}
V_{t}:=e^{-r(T-t)} V^{A m}\left(t, S_{t}\right), \\
\left(r e s p .-e^{-r(T-t)} \operatorname{ess~sup}_{\nu \in \mathscr{F}_{t, T}}^{G}\left[-f_{\nu}\right]\right)
\end{gathered}
$$

is the ask (resp., bid) price process against $\xi$.

Proof. The value function for the optimal stop time $V^{A m}\left(t, S_{t}\right)$ is a G-supermartingale; it is easily to check that $e^{-r t} V_{t}$ is G-supermartingale. By G-Doob-Meyer decomposition Theorem 24

$$
e^{-r t} V_{t}=M_{t}-\bar{C}_{t}
$$

where $M_{t}$ is a G-martingale and $\bar{C}_{t}$ is an increasing process with $\bar{C}_{0}=0$. By G-martingale representation theorem $[14,21]$

$$
M_{t}=E^{G}\left[M_{T}\right]+\int_{0}^{t} \eta_{s} d \widetilde{B}_{t}-K_{t}
$$

where $\eta_{s} \in H_{G}^{1}(0, T),-K_{t}$ is a G-martingale, and $K_{t}$ is an increasing process with $K_{0}=0$. From the above equation, we have

$$
e^{-r t} V_{t}=E^{G}\left[M_{T}\right]+\int_{0}^{t} \eta_{s} d \widetilde{B}_{t}-\left(K_{t}+\bar{C}_{t}\right)
$$

hence $\left(V_{t}, e^{r t} \eta_{t}, \int_{0}^{t} e^{r s} d\left(\bar{C}_{s}+K_{s}\right) d s\right)$ is a superhedging strategy.

Assume that $\left(Y_{t}, \pi_{t}, C_{t}\right)$ is a superhedging strategy against $\xi$; then

$$
e^{-r t} Y_{t}=e^{-r T} \xi-\int_{t}^{T} \pi_{t} d \widetilde{B}_{t}+C_{t}
$$

Taking conditional G-expectation on the both sides of (84) and noticing that the process $C_{t}$ is an increasing process with $C_{0}=0$, we derive

$$
e^{-r t} Y_{t} \geq E_{t}^{G}\left[e^{-r T} \xi\right]
$$

which implies that

$$
\begin{aligned}
Y_{t} & \geq E_{t}^{G}\left[e^{-r(T-t)} \xi\right] \geq E_{t}^{G}\left[e^{-r(T-t)} \underset{\nu \in \mathscr{F}_{T, T}}{\operatorname{ess} \sup _{\nu}}\left[f_{\nu}\right]\right] \\
& \geq e^{-r(T-t)} \underset{\nu \in \mathscr{F}_{T, T}}{\operatorname{ess} \sup _{t}}\left[f_{\nu}\right] \geq e^{-r(T-t)} \underset{\nu \in \mathscr{F}_{t, T}}{\operatorname{ess} \sup _{t}} E_{t}^{G}\left[f_{\nu}\right] \\
& =V_{t}
\end{aligned}
$$

from which we prove that $V_{t}=e^{-r(T-t)} V^{A m}\left(t, S_{t}\right)$ is the ask price against the claim $\xi$ at time $t$. Similarly we can prove that $-e^{-r(T-t)}$ ess $\sup _{\nu \in \mathscr{F}_{t, T}} E_{t}^{G}\left[-f_{\nu}\right]$ is the bid price against the claim $\xi$ at time $t$. 


\section{Free Boundary and Optimal Stopping Problems}

For given $t \in[0, T], x \in R^{d}$, and $d=1$, the G-asset price system (58) of the risky asset can be rewritten as follows:

$$
\begin{aligned}
d S_{u}^{t, x} & =S_{u}^{t, x}\left(r d t+d \widetilde{B}_{t}\right), \\
S_{t}^{t, x} & =x .
\end{aligned}
$$

We define the following deterministic function:

$$
u^{a}(t, x):=e^{-r(T-t)} V^{A m}\left(t, S_{t}^{t, x}\right)
$$

where

$$
V^{A m}\left(t, S_{t}^{t, x}\right)=\underset{v \in \mathscr{F}_{t, T}}{\operatorname{ess} \sup } E_{t}^{G}\left[f\left(S_{\nu}^{t, x}\right)\right]
$$

From Theorem 31 the price of an American option with expiry date $T$ and payoff function $f$ is the value function of the optimal stopping problem:

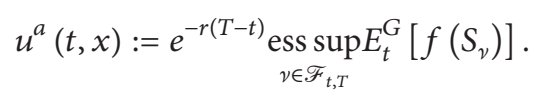

We define operator $L$ as follows:

$$
L u=G\left(D^{2} u\right)+r D u+\partial_{t} u
$$

where $G(\cdot)$ is the sublinear function defined by (9). We consider the free boundary problem

$$
\begin{aligned}
\mathscr{L} u & :=\max \{L u-r u, f-u\}=0, \quad \text { in }[0, T] \times R, \\
u(T, \cdot) & =f(T, \cdot), \quad \text { in } R .
\end{aligned}
$$

Denote

$$
\mathcal{S}_{T}:=[0, T] \times R,
$$

for $p \geq 1$

$$
\mathcal{S}^{p}\left(\mathcal{S}_{T}\right):=\left\{u \in L^{p}\left(\mathcal{S}_{T}\right): D^{2} u, D u, \partial_{t} u \in L^{p}\left(\mathcal{S}_{T}\right)\right\}
$$

And, for any compact subset $D$ of $\mathcal{S}_{T}$, we denote $\mathcal{S}_{\text {loc }}^{p}(D)$ as the space of functions $u \in \mathcal{S}^{p}(D)$.

Definition 32. A function $u \in \mathcal{S}_{\mathrm{loc}}^{1}\left(\mathcal{S}_{T}\right) \cap C(R \times[0, T])$ is a strong solution of problem (92) if $\mathscr{L} u=0$ almost everywhere in $\mathcal{S}_{T}$ and it attains the final datum pointwisely. A function $u \in \mathcal{S}_{\mathrm{loc}}^{1}\left(\mathcal{S}_{T}\right) \cap C(R \times[0, T])$ is a strong supersolution of problem (92) if $\mathscr{L} u \leq 0$.

We will prove the following existence results.

Theorem 33. If there exists a strong supersolution $\bar{u}$ of problem (92) then there also exists a strong solution $u$ of (92) such that $u \leq \bar{u}$ in $\mathcal{S}_{T}$. Moreover $u \in \mathcal{S}_{\text {loc }}^{p}\left(\mathcal{S}_{T}\right)$ for any $p \geq 1$ and consequently, by the embedding theorem we have $u \in$ $C_{B, l o c}^{1, \alpha}\left(\mathcal{S}_{T}\right)$ for any $\alpha \in[0,1]$.
Theorem 34. Let $u$ be a strong solution to the free boundary problem (92) such that

$$
|u(t, x)| \leq C e^{\lambda|x|^{2}}, \quad(t, x) \in \mathcal{S}_{T}
$$

form some constants $C, \lambda$ with $\lambda$ sufficiently small so that

$$
E^{G}\left[\exp \left(\lambda \sup _{t \leq u \leq T}\left|S_{u}^{t, x}\right|^{2}\right)\right]<\infty
$$

holds. Then we have

$$
u(t, x)=e^{-r(T-t)} \underset{v \in \mathscr{F}_{t, T}}{\operatorname{ess} \sup _{t}} E_{t}^{G}\left[f\left(S_{\nu}\right)\right]
$$

that is, the solution of the free boundary problem is the value function of the optimal stopping problem. In particular such a solution is unique.

5.1. Proof of Theorem 34. We employ a truncation and regularization technique to exploit the weak interior regularity properties of $u$; for $R>0$ we set for $R>0, B_{R}:=\left\{x \in R^{d} \mid\right.$ $|x|<R\}$, and, for $x \in B_{R}$ denoting by $\tau_{R}$ the first exit time of $S_{u}^{t, x}$ from $B_{R}$, it is easy check that $E^{G}\left[\tau_{R}\right]$ is finite. As a first step we prove the following result: for every $(t, x) \in[0, T] \times B_{R}$ and $\tau \in \mathscr{F}_{t, T}$ such that $\tau \in\left[t, \tau_{R}\right]$, it holds that

$$
u(t, x)=E^{G}\left[u\left(\tau, S_{\tau}^{t, x}\right)\right]-E^{G}\left[\int_{t}^{\tau} L u\left(s, S_{s}^{t, x}\right) d s\right] .
$$

For fixed, positive, and small enough $\varepsilon$, we consider a function $u^{\varepsilon, R}$ on $R^{d+1}=R^{2}$ with compact support and such that $u^{\varepsilon, R}=u$ on $[t, T-\varepsilon] \times B_{R}$. Moreover we denote by $\left(u^{\varepsilon, R, n}\right)_{n \in N}$ a regularizing sequence obtained by convolution of $u^{\varepsilon, R}$ with the usual mollifiers; then for any $p \geq 1$ we have $u^{\varepsilon, R, n} \in$ $\mathcal{S}^{p}\left(R^{2}\right)$ and

$$
\lim _{n \rightarrow \infty}\left\|L u^{\varepsilon, R, n}-L u^{\varepsilon, R}\right\|_{L^{p}\left([t, T-\varepsilon] \times B_{R}\right)}=0 .
$$

By G-Itô formula we have

$$
\begin{aligned}
u^{\varepsilon, R, n}\left(\tau, S_{\tau}^{t, x}\right)= & u^{\varepsilon, R, n}(t, x)+\frac{1}{2} \int_{t}^{\tau} D^{2} u^{\varepsilon, R, n} d\langle B\rangle_{s} \\
& +\int_{t}^{\tau} r D u^{\varepsilon, R, n} d s+\int_{t}^{\tau} \partial_{s} u^{\varepsilon, R, n} d s \\
& +\int_{t}^{\tau} D u^{\varepsilon, R, n} d B_{s},
\end{aligned}
$$

which implies that

$$
E^{G}\left[u^{\varepsilon, R, n}\left(\tau, S_{\tau}^{t, x}\right)\right]=u^{\varepsilon, R, n}(t, x)+\int_{t}^{\tau} L u^{\varepsilon, R, n} d s .
$$

We have

$$
\lim _{n \rightarrow \infty} u^{\varepsilon, R, n}(t, x)=u^{\varepsilon, R}(t, x)
$$

and, by dominated convergence,

$$
\lim _{n \rightarrow \infty} E^{G}\left[u^{\varepsilon, R, n}\left(\tau, S_{\tau}^{t, x}\right)\right]=E^{G}\left[u^{\varepsilon, R}\left(\tau, S_{\tau}^{t, x}\right)\right] .
$$


We have

$$
\begin{aligned}
& \mid E^{G}\left[\int_{t}^{\tau} L u^{\varepsilon, R, n}\left(s, S_{s}^{t, x}\right) d s\right] \\
& -E^{G}\left[\int_{t}^{\tau} L u^{\varepsilon, R}\left(s, S_{s}^{t, x}\right) d s\right] \mid \\
& \quad \leq E^{G}\left[\int_{t}^{\tau}\left|L u^{\varepsilon, R, n}\left(s, S_{s}^{t, x}\right)-L u^{\varepsilon, R}\left(s, S_{s}^{t, x}\right)\right| d s\right] ;
\end{aligned}
$$

by sublinear expectation representation theorem (see [14]) there exists a family of probability space $Q$, such that

$$
\begin{aligned}
E^{G} & {\left[\int_{t}^{\tau}\left|L u^{\varepsilon, R, n}\left(s, S_{s}^{t, x}\right)-L u^{\varepsilon, R}\left(s, S_{s}^{t, x}\right)\right| d s\right] } \\
= & \underset{P \in Q}{\operatorname{ess} \sup _{P}} E_{P}\left[\int_{t}^{\tau} \mid L u^{\varepsilon, R, n}\left(s, S_{s}^{t, x}\right)\right. \\
& \left.-L u^{\varepsilon, R}\left(s, S_{s}^{t, x}\right) \mid d s\right] .
\end{aligned}
$$

Since $\tau \leq \tau_{R}$

$$
\begin{aligned}
& \underset{P \in Q}{\operatorname{ess} \sup } E_{P}\left[\int_{t}^{\tau}\left|L u^{\varepsilon, R, n}\left(s, S_{s}^{t, x}\right)-L u^{\varepsilon, R}\left(s, S_{s}^{t, x}\right)\right| d s\right] \\
& \leq \underset{P \in Q}{\operatorname{ess} \sup _{P}} E_{P}\left[\int_{t}^{T-\varepsilon}\left|L u^{\varepsilon, R, n}(s, y)-L u^{\varepsilon, R}(s, y)\right|\right. \\
& \left.\cdot I_{\left|S_{s}^{t, x}\right| \leq B_{R}} d s\right] \leq \underset{P \in Q}{\operatorname{ess} \sup } \int_{t}^{T-\varepsilon} \int_{B_{R}} \mid L u^{\varepsilon, R, n}(s, y) \\
& -L u^{\varepsilon, R}(s, y) \mid \Gamma_{P}(t, x ; s, y) d y d s
\end{aligned}
$$

where $\Gamma_{P}(t, x ; \cdot, \cdot) \in L^{\bar{q}}\left([t, T] \times B_{R}\right)$, for some $\bar{q}>1$, is the transition density of the solution of

$$
d X_{s}^{t, x}=X_{s}^{t, x}\left(r d s+\sigma_{s, P} d W_{s, P}\right),
$$

where $W_{s, P}$ is Wiener process in probability space $\left(\Omega_{t}, P\right.$, $\left.\mathscr{F}^{P}, \mathscr{F}_{t}^{P}\right)$ and $\sigma_{s, P}$ is adapted process such that $\sigma_{s, P} \in[\underline{\sigma}, \bar{\sigma}]$. By Hölder inequality, we have $(1 / \bar{p}+1 / \bar{q}=1)$

$$
\begin{aligned}
& \int_{t}^{T-\varepsilon} \int_{B_{R}}\left|L u^{\varepsilon, R, n}(s, y)-L u^{\varepsilon, R}(s, y)\right| \\
& \quad \cdot \Gamma_{P}(t, x ; s, y) d y d s \leq \| L u^{\varepsilon, R, n}(s, y) \\
& \quad-L u^{\varepsilon, R}(s, y)\left\|_{L^{\bar{q}}\left([t, T] \times B_{R}\right)}\right\| \Gamma_{P}(t, x ; s, y) \|_{L^{\bar{p}}\left([t, T] \times B_{R}\right)},
\end{aligned}
$$

and then we obtain that

$$
\begin{gathered}
\lim _{n \rightarrow \infty} E^{G}\left[\int_{t}^{\tau} L u^{\varepsilon, R, n}\left(s, S_{s}^{t, x}\right)\right] \\
=E^{G}\left[\int_{t}^{\tau} L u^{\varepsilon, R}\left(s, S_{s}^{t, x}\right)\right] .
\end{gathered}
$$

This concludes the proof of (98), since $u^{\varepsilon, R}=u$ on $[t, T-\varepsilon] \times$ $B_{R}$ and $\varepsilon>0$ is arbitrary.
Since $L u \leq 0$, we have for any $\tau \in \mathscr{F}_{t, T}$

$$
E^{G} \int_{t}^{\tau} \operatorname{Lu}\left(s, S_{s}^{t, x}\right) d s \leq 0
$$

we infer from (98) that

$$
u(t, x) \geq E^{G}\left[u\left(\tau \wedge \tau_{R}, S_{\tau \wedge \tau_{R}}^{t, x}\right)\right] .
$$

Next we pass to the limit as $R \rightarrow+\infty$ : we have

$$
\lim _{R \rightarrow+\infty} \tau \wedge \tau_{R}=\tau,
$$

and by the growth assumption (95)

$$
\left|u\left(\tau \wedge \tau_{R}, S_{\tau \wedge \tau_{R}}^{t, x}\right)\right| \leq C \exp \left(\lambda \sup _{t \leq s \leq T}\left|S_{s}^{t, x}\right|^{2}\right) .
$$

As $R \rightarrow+\infty$

$$
u(t, x) \geq E^{G}\left[u\left(\tau, S_{\tau}^{t, x}\right)\right] \geq E^{G}\left[f\left(\tau, S_{\tau}^{t, x}\right)\right] .
$$

This shows that

$$
u(t, x) \geq \sup _{\tau \in \mathscr{F}_{t, T}} E^{G}\left[f\left(\tau, S_{\tau}^{t, x}\right)\right] .
$$

We conclude the proof by putting

$$
\tau_{0}=\inf \left\{s \in[t, T] \mid u\left(s, S_{s}^{t, x}\right)=f\left(s, S_{s}^{t, x}\right)\right\} .
$$

Since $L u=0$ a.e., where $u>\phi$, it holds

$$
E^{G}\left[\int_{t}^{\tau_{0} \wedge \tau_{R}} L u\left(s, S_{s}^{t, x}\right) d s\right]=0
$$

and from (98) we derive that

$$
u(t, x)=E^{G}\left[u\left(\tau_{0} \wedge \tau_{R}, S_{\tau_{0} \wedge \tau_{R}}^{t, x}\right)\right] .
$$

Repeating the previous argument to pass to the limit in $R$, we obtain

$$
u(t, x)=E^{G}\left[u\left(\tau_{0}, S_{\tau_{0}}^{t, x}\right)\right]=E^{G}\left[f\left(\tau_{0}, S_{\tau_{0}}^{t, x}\right)\right] .
$$

Therefore, we finish the proof.

5.2. Free Boundary Problem. Here we consider the free boundary problem on a bounded cylinder. We denote the bounded cylinders as the form $[0, T] \times H_{n}$, where $\left(H_{n}\right)$ is an increasing covering of $R^{d}(d=1)$. We will prove the existence of a strong solution to problem

$$
\begin{aligned}
\max \{L u, f-u\} & =0, \quad \text { in } H(T):=[0, T] \times H, \\
\left.u\right|_{\partial_{P} H(T)} & =f,
\end{aligned}
$$

where $H$ is a bounded domain of $R^{d}$ and

$$
\partial_{P} H(T):=\partial H(T) \backslash(\{T\} \times H)
$$

is the parabolic boundary of $H(T)$.

We assume the following condition on the payoff function. 
Assumption 35. The payoff function $\xi=f\left(S_{u}^{t, x}\right)$ has the following assumption expressed by the sublinear function:

$$
-G\left(-D^{2} f\right) \geq c \quad \text { in } H
$$

where $G(\cdot)$ is the sublinear function defined by (9).

Theorem 36. One assumes assumption 5.1 holds. Problem (120) has a strong solution $u \in \mathcal{S}_{l o c}^{1}(H(T)) \cap C(\overline{H(T)})$. Moreover $u \in \mathcal{S}_{\text {loc }}^{p}(H(T))$ for any $p>1$.

Proof. The proof is based on a standard penalization technique (see [18]). We consider a family $\left(\beta_{\varepsilon}\right)_{\varepsilon \in[0,1]}$ of smooth functions such that, for any $\varepsilon$, function $\beta_{\varepsilon}$ is increasing bounded on $R$ and has bounded first order derivative, such that

$$
\begin{aligned}
\beta_{\varepsilon}(s) & \leq \varepsilon, \quad s>0, \\
\lim _{\varepsilon \rightarrow 0} \beta_{\varepsilon}(s) & =-\infty, \quad s<0 .
\end{aligned}
$$

We denote by $f^{\delta}$ the regularization of $f$ and consider the following penalized and regularized problem and denote the solution as $u_{\varepsilon, \delta}$

$$
\begin{aligned}
L u & =\beta_{\varepsilon}\left(u-f^{\delta}\right), \quad \text { in } H(T), \\
\left.u\right|_{\partial_{P} H(T)} & =f^{\delta} ;
\end{aligned}
$$

Lions [24], Krylov [25], and Nisio [26] prove that problem (124) has a unique viscosity solution $u_{(\varepsilon, \delta)} \in C^{2, \alpha}(\overline{H(T)}) \cap$ $C(\overline{H(T)})$ with $\alpha \in[0,1]$.

Next, we firstly prove the uniform boundedness of the penalization term:

$$
\left|\beta_{\varepsilon}\left(u_{\varepsilon, \delta}-f^{\delta}\right)\right| \leq c, \quad \text { in } H(T),
$$

with $c$ independent of $\varepsilon$ and $\delta$.

By construction $\beta_{\varepsilon} \leq \varepsilon$, it suffices to prove the lower bound in (125). By continuity, $\beta_{\varepsilon}\left(u_{\varepsilon, \delta}-f^{\delta}\right)$ has a minimum $\zeta$ in $\overline{H(T)}$ and we may suppose

$$
\beta_{\varepsilon}\left(u_{\varepsilon, \delta}(\zeta)-f^{\delta}(\zeta)\right) \leq 0
$$

otherwise we prove the lower bound. If $\zeta \in \partial_{P} H(T)$ then

$$
\beta_{\varepsilon}\left(u_{\varepsilon, \delta}(\zeta)-f^{\delta}(\zeta)\right)=\beta_{\varepsilon}(0)=0
$$

On the other hand, if $\zeta \in H(T)$, then we recall that $\beta_{\varepsilon}$ is increasing and consequently $u_{(\varepsilon, \delta)}-f^{\delta}$ also has a (negative) minimum in $\zeta$. Thus, we have

$$
L u_{\varepsilon, \delta}(\zeta)-L f^{\delta}(\zeta) \geq 0 \geq u_{\varepsilon, \delta}(\zeta)-f^{\delta}(\zeta)
$$

By Assumption 35 on $f$, we have that $L f^{\delta}(\zeta)$ is bounded uniformly in $\delta$. Therefore, by (128), we deduce

$$
\beta_{\varepsilon}\left(u_{(\varepsilon, \delta)}(\zeta)-f^{\delta}(\zeta)\right)=L u_{(\varepsilon, \delta)}(\zeta) \geq L f^{\delta}(\zeta) \geq c
$$

where $c$ is a constant independent of $\varepsilon, \delta$ and this proves (125).

Secondly, we use the $\mathcal{S}^{p}$ interior estimate combined with (125), to infer that, for every compact subset $D$ in $H(T)$ and $p \geq 1$, the norm $\left\|u_{\varepsilon, \delta}\right\|_{\mathcal{S}^{p}(D)}$ is bounded uniformly in $\varepsilon$ and $\delta$. It follows that $\left(u_{\varepsilon, \delta}\right)$ converges as $\varepsilon, \delta \rightarrow 0$ weakly in $\mathcal{S}^{p}$ on compact subsets of $H(T)$ to a function $u$. Moreover

$$
\limsup _{\varepsilon, \delta} \beta_{\varepsilon}\left(u_{\varepsilon, \delta}-f^{\delta}\right) \leq 0
$$

so that $L u \leq f$ a.e. in $H(T)$. On the other hand, $L u=f$ a.e. in set $\{u>f\}$.

Finally, it is straightforward to verify that $u \in C(\overline{H(T)})$ and assumes the initial-boundary conditions, by using standard arguments based on the maximum principle and barrier functions.

Proof of Theorem 33. The proof of Theorem 33 about the existence theorem for the free boundary problem on unbounded domains is similar to [27] by using Theorem 36 about the existence theorem for the free boundary problem on the regular bounded cylindrical domain.

\section{Conflict of Interests}

The author declares that there is no conflict of interests regarding the publication of this paper.

\section{References}

[1] H. P. McKean Jr., "Appendix: a free boundary problem for the heat equation arising from a problem in mathematical economics," Industrial Management Review, vol. 6, pp. 32-39, 1965.

[2] A. Bensoussan, "On the theory of option pricing," Acta Applicandae Mathematicae, vol. 2, no. 2, pp. 139-158, 1984.

[3] F. Black and M. Scholes, "The pricing of options and corporate liabilities," Journal of Political Economy, vol. 81, no. 3, pp. 637$659,1973$.

[4] I. Karatzas, "On the pricing of American options," Applied Mathematics \& Optimization, vol. 17, no. 1, pp. 37-60, 1988.

[5] N. EL Karoui and I. Karatzas, "Integration of the optimal risk in a stopping problem with absorption," in Séminaire de Probabilités XXIII, vol. 1372 of Lecture Notes in Mathematics, pp. 405-420, Springer, Berlin, Germany, 1989.

[6] N. EL Karoui and I. Karatzas, "A new approach to the Skorohod problem and its applications," Stochastics and Stochastic Reports, vol. 34, pp. 57-82, 1991.

[7] J. L. Doob, Stochastic Processes, John Wiley \& Sons, New York, NY, USA, 1953.

[8] P. A. Meyer, "A decomposition theorem for supermartingales," Illinois Journal of Mathematics, vol. 6, pp. 193-205, 1962.

[9] P.-A. Meyer, "Decomposition of supermartingales: the uniqueness theorem," Illinois Journal of Mathematics, vol. 7, pp. 1-17, 1963. 
[10] D. O. Kramkov, "Optional decomposition of supermartingales and hedging contingent claims in incomplete security markets," Probability Theory and Related Fields, vol. 105, no. 4, pp. 459479, 1996.

[11] R. Frey, "Superreplication in stochastic volatility models and optimal stopping," Finance and Stochastics, vol. 4, no. 2, pp. 161$187,2000$.

[12] F. Knight, Risk, Uncertainty, and Profit, Houghton Mifflin Harcourt, Boston, Mass, USA, 1921.

[13] S. G. Peng, "Survey on normal distributions, central limit theorem, Brownian motion and the related stochastic calculus under sublinear expectations," Science in China, Series A: Mathematics, vol. 52, no. 7, pp. 1391-1411, 2009.

[14] S. G. Peng, "Nonlinear expectations and stochastic calculus underuncertainty," http://arxiv.org/abs/1002.4546.

[15] L. G. Epstein and S. Ji, "Ambiguous volatility and asset pricing in continuous time," Review of Financial Studies, vol. 26, no. 7, pp. 1740-1786, 2013.

[16] J. Vorbrink, "Financial markets with volatility uncertainty," Journal of Mathematical Economics, vol. 53, pp. 64-78, 2014.

[17] W. Chen, "G-consistent price system and bid-ask pricing for European contingent claims underKnightian uncertainty," http://arxiv.org/abs/1308.6256.

[18] A. Friedman, Variational Principles and Free-Boundary Problems, Pure and Applied Mathematics, John Wiley \& Sons, New York, NY, USA, 1982.

[19] X. P. Li and S. G. Peng, "Stopping times and related Itô's calculus with G-Brownian motion," Stochastic Processes and Their Applications, vol. 121, no. 7, pp. 1492-1508, 2011.

[20] M. Hu and S. Peng, "Extended conditional G-expectations and related stoppingtimes," http://arxiv.org/abs/1309.3829.

[21] Y. Z. Song, "Some properties on G-evaluation and its applications to G-martingale decomposition," Science China Mathematics, vol. 54, no. 2, pp. 287-300, 2011.

[22] W. Chen, "Time consistent G-expectationand bid-ask dynamic pricing mechanisms for contingent claims under uncertainty," http://arxiv.org/abs/1111.4298v1.

[23] M. Hu, S. Ji, S. Peng, and Y. Song, "Comparison theorem, Feynman-Kacformula and Girsanov transformation for BSDEs driven by G-Brownian motion," http://arxiv.org/abs/1212 $.5403 \mathrm{vl}$.

[24] P.-L. Lions, "Optimal control of diffusion processes and HJB equations part II: viscosity and uniqueness," Communications in Partial Differential Equations, vol. 8, no. 11, pp. 1229-1276, 1983.

[25] N. V. Krylov, Controlled Diffusion Processes, Springer, New York, NY, USA, 1980.

[26] M. Nisio, Stochastic Control Theory, vol. 9 of ISI Lectures Notes, Tata Institute, MacMillan India, 1981.

[27] M. Di Francesco, A. Pascucci, and S. Polidoro, "The obstacle problem for a class of hypoelliptic ultraparabolic equations," Proceedings of the Royal Society A: Mathematical, Physical and Engineering Sciences, vol. 464, no. 2089, pp. 155-176, 2008. 


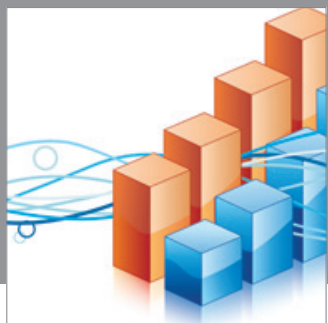

Advances in

Operations Research

mansans

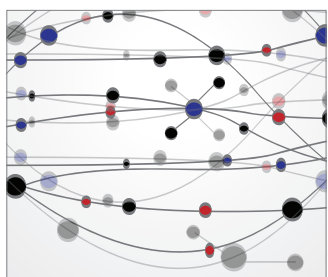

The Scientific World Journal
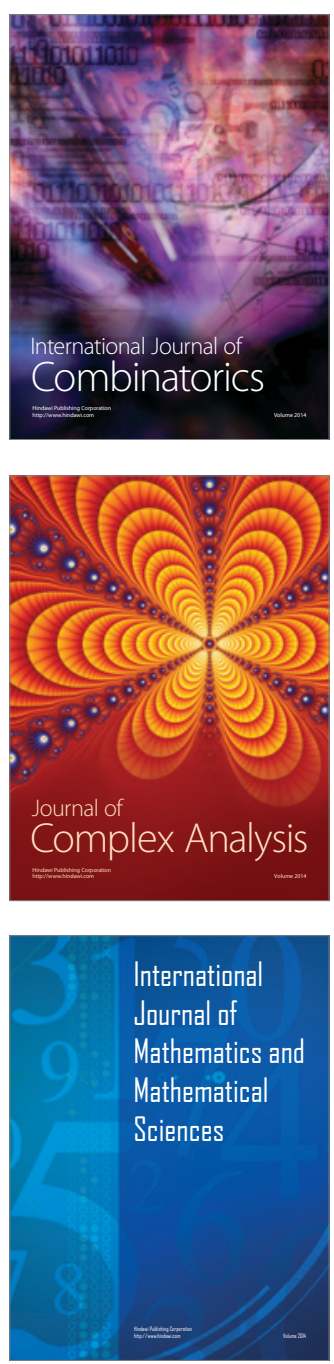
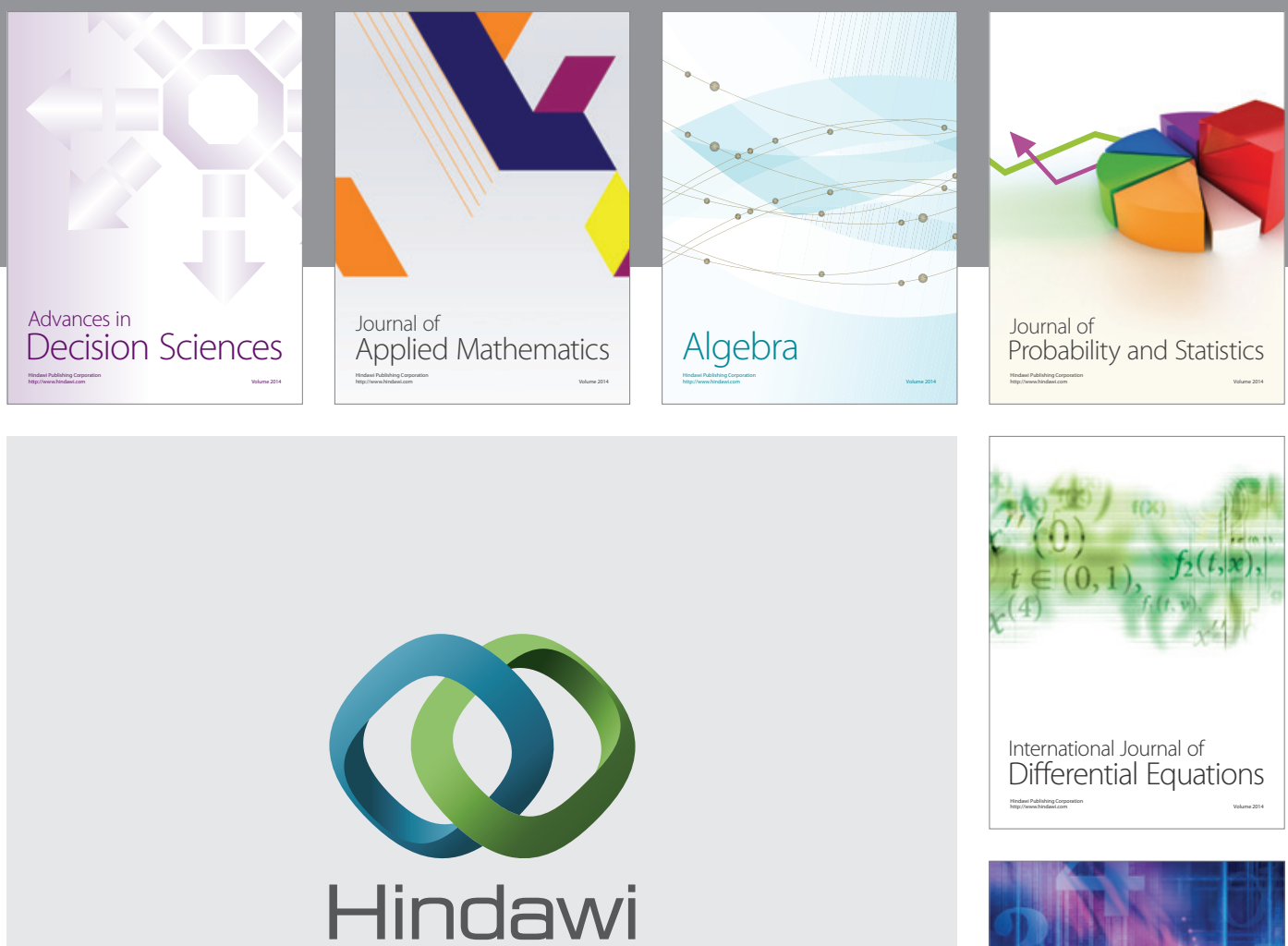

Submit your manuscripts at http://www.hindawi.com
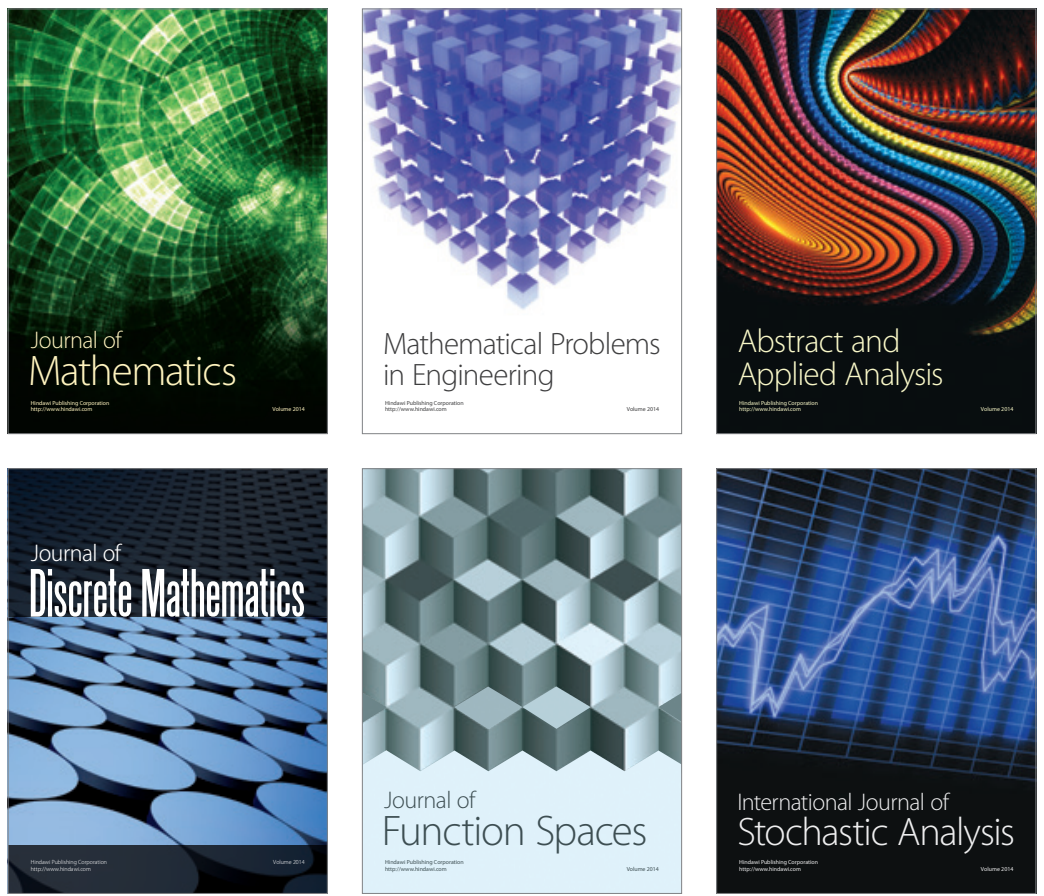

Journal of

Function Spaces

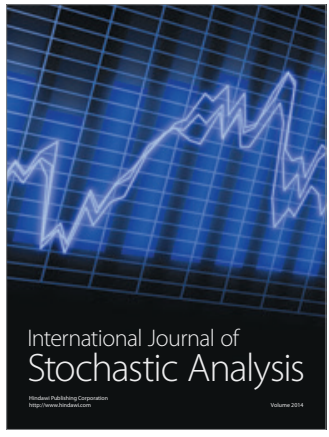

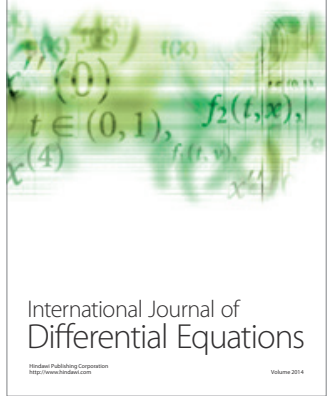
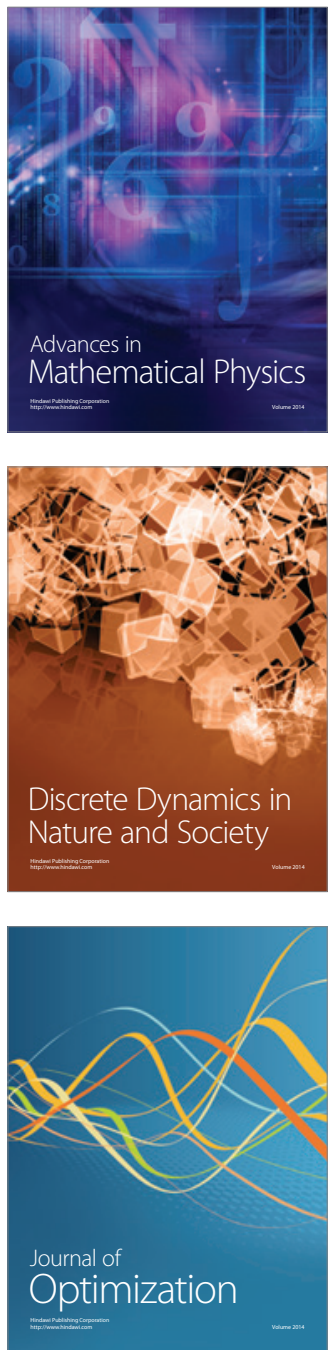